\title{
In-orbit Performance of the Silicon-Tungsten Tracker of the DAMPE Mission
}

\author{
X. Wu, for the DAMPE Collaboration \\ Département de Physique Nucléaire et Corpulsculaire, University of Geneva, Geneva, Switzerland
}

The DArk Matter Particle Explorer (DAMPE) is a high energy astroparticle satellite mission designed to detect electron, photon and cosmic rays with high precision for Dark Matter search, cosmic ray flux and composition measurement and gamma-ray astronomy. One of the key components of the DAMPE payload is the Silicon-Tungsten Tracker (STK), consisting of 6 tracking planes, each plane is made of 2 orthogonal layers of single-sided silicon micro-strip detectors. Three layers of $1 \mathrm{~mm}$ thick tungsten plates are interleaved with the tracking planes to serve as photon converter. Besides precise track reconstruction for charge particles and converted photons, the STK will also measure the charge of the incoming cosmic ray, and provide pre-shower information to improve particle identification.

After intensive design, prototyping, test and production efforts by the STK collaboration, the construction of the STK has been completed in April 2015 and successfully integrated into the DAMPE payload in June 2015, after passing the environmental acceptance test. The DAMPE satellite was launched on December $17^{\text {th }} 2015$.

After the launch the STK has been commissioned rapidly in orbit and has ben functioning extremely well since, which allows it to play a key role in the first physics results coming out of the DAMPE collaboration. In this contribution, a brief overview on the development, qualification and beam tests of the STK Engineering and Qualification Model and the Flight Model will be provided. Then the STK in-orbit calibration and performance will be presented in details, including the noise behavior, the thermal and mechanical stability, the alignment procedure, the position resolution, and the tracking efficiency.

The 35th International Cosmic Ray Conference - ICRC 2017-

$12-20$ July, 2017

BEXCO, BUSAN, KOREA 


\section{Introduction}

The DArk Matter Particle Explorer (DAMPE)[1] is a high energy astroparticle satellite mission designed to detect high energy gamma rays and cosmic rays with high precision. The main science objectives are 1) the search for Dark Matter decay or annihilation signatures, 2) the measurement of cosmic ray flux and composition, and 3) gamma-ray astronomy. DAMPE is one of the five scientific space science missions planned within the framework of the Strategic Pioneer Program on Space Science of the Chinese Academy of Sciences (CAS). The design, prototyping, production, test and qualification of the DAMPE payload has been carried out by collaborating institutes ${ }^{1}$ from China, Switzerland and Italy.

The performance goal of the DAMPE detector is to measure precisely the electron and photon spectrum between $5 \mathrm{GeV}$ to $10 \mathrm{TeV}$, and cosmic ray flux and chemical composition between $10 \mathrm{GeV}$ to above $100 \mathrm{TeV}$, with reasonably large geometrical acceptances $\left(\sim 0.3 \mathrm{~m}^{2} \mathrm{sr}\right.$ for electrons/photons), using the technologies of a thick total absorption calorimeter, and a precise tracker with integrated photon converters. The DAMPE detector, as shown in Figure 1, consists of 4 subsystems: the plastic scintillator strips detector (PSD), the silicon-tungsten tracker-converter (STK), the BGO imaging calorimeter (BGO), and the neutron detector (NUD).

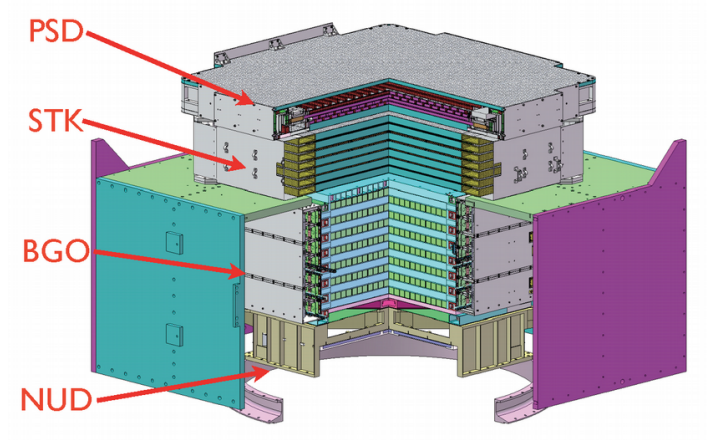

Figure 1: Sketch of the DAMPE detector

\section{The Silicon-Tungsten Tracker}

Silicon micro-strip detector is a proven space technology that is used successfully in space experiments, eg. PAMELA, AGILE, Fermi and AMS. The tracker-converter concept, in which tungsten foils are inserted between silicon tacking layers, has been employed in AGILE and Fermi and proved to be an excellent technique to detect high-energy photons with high efficiency and precision. An incoming photon converts to an electron-positron pair in the tungsten converter, which can then be detected by subsequent silicon layers. Multiple scattering of charged particles can be mitigated by spreading the tungsten to several foils, and for highenergy particles $(>5 \mathrm{GeV})$, the effect is negligible for tungsten foils of a few milimeters thickness $\left(\sim 0.08^{\circ}\right.$ angular smearing for $5 \mathrm{GeV}$ particles after $1 \mathrm{~mm}$ tungsten). The STK[2] is made of 6 tracking planes each consists of two layers of single-sided silicon strip detectors measuring the two orthogonal views perpendicular to the pointing direction of the apparatus.

${ }^{1}$ PMO-USTC-IMP-IHEP-NSSC-UNIGE-PERUGIA-BARI-LECCE 
Three layers of tungsten plates of $1 \mathrm{~mm}$ thick are inserted in front of tracking layer 2, 3 and 4 for photon conversion. Figure 2 shows an exploded view of the STK internal structure (left panel) and a picture of the STK Flight Model during the final assembly (right panel).
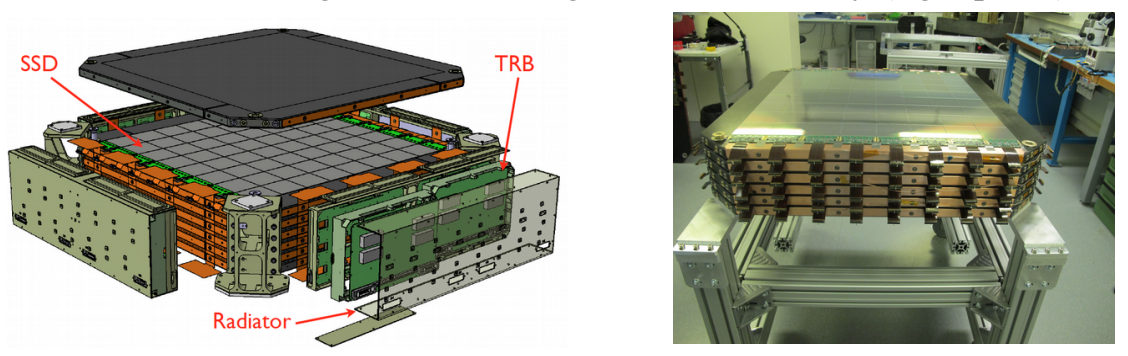

Figure 2: Exploded view of the STK (left). STK before the assembly of the last tray(right).

The STK uses single-sided AC-coupled silicon micro-strip detectors. The sensor is $9.5 \mathrm{~cm}$ by $9.5 \mathrm{~cm}$ in size, $320 \mu \mathrm{m}$ thick, and segmented into 768 strips with a $121 \mu \mathrm{m}$ pitch. Only every other strip is readout but since analogue readout is used the position resolution is better than $80 \mu \mathrm{m}$ for most incident angles, thanks to the charge division of floating strips. The photon angular resolution is expected to be around $0.2^{\circ}$ at $10 \mathrm{GeV}$. The high dynamic range of the analog readout electronics of the STK allows to measure the charge of the incident cosmic rays with high precision. The full tracker uses 768 sensors, equivalent to a total silicon area of $\sim 7 \mathrm{~m}^{2}$. The total number of readout channels is 73,728 . The power consumption of each ladder is about $116 \mathrm{~mW}$ and the total for the STK is about $90 \mathrm{~W}$.

\section{On-ground tests and calibrations of the STK}

The main design concepts of the mechanical support structure and thermal management have been qualified with the EQM in a series of space environment qualification tests, including vibration, acceleration, shock, thermal cycling and thermal vacuum tests. The EQM is a full size tracker model that has the same thermal, mechanical and data interface to the satellite as the final Flight Model (FM), but with only 26 functioning silicon ladders, the remaining 166 being mechanical ladders with the same mechanical and thermal properties as the real ladders. The EQM has also been integrated into the DAMPE prototype full detector and tested extensively with particle beams at CERN PS and SPS during 2014 and 2015. Data with protons, electrons, muons, photons and nuclear fragments have been taken and are being analysed to assess the detector performance.

The final Flight Model has been calibrated extensively with cosmic muons on ground during the final satellite integration, qualification and transportation. After $\sim 6$ months of intensive manipulations and tests the STK remained in excellent quality. At the end of the satellite integration test, the percentage of noisy channels (noise $>5$ ADC counts) remained below $0.4 \%$ (see Figure 3). For comparison, the noise distribution in-orbit, 10 months after the launch, is also shown: the amount of noisy channels is slightly less $(0.3 \%)$, and the bulk of the noise is lower, due to the stabilisation process, and a colder operation temperature in orbit (see the next Chapter).

The large amount of cosmic data collected has been used to align the STK in order to correct for displacement and rotation of the SSDs with respect to the nominal position, and to monitor the stability of the STK mechanical structure. As a result of the alignment, the spatial resolution is below $80 \mu \mathrm{m}$ within the angular acceptance of the STK (i.e. incidence angle $<60^{\circ}$ ) 
and below $60 \mu \mathrm{m}$ for particle incidence angles within $40^{\circ}$. This result is in agreement with the spatial resolution measurements obtained in test beam campaigns at CERN SPS on a single ladder $[2,3]$.
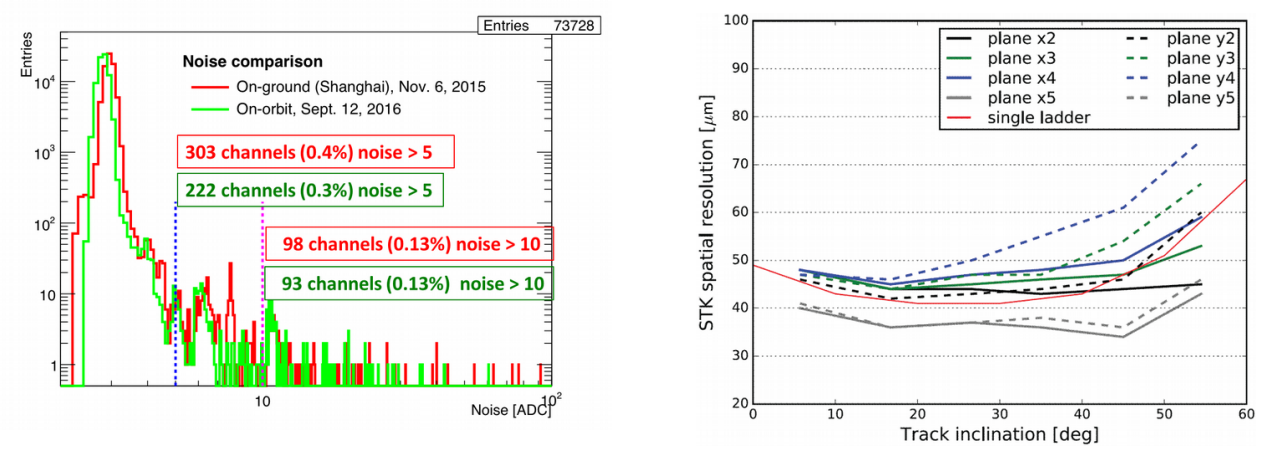

Figure 3: Noise distribution of the 73728 STK channels, on-ground and in-orbit (left); Spatial resolution for different STK planes as a function of particle incident angle for cosmic rays data at ground. The results obtained from a test beam campaign on single ladder are also shown as reference (right).

\section{The commissioning and the performance of the STK in-orbit}

On Dec. $17^{\text {th }} 2015$ DAMPE was successfully launched into a sun-synchronous orbit at the altitude of $500 \mathrm{~km}$. Three days after the launch the STK was powered on, and four days later, the high voltage of the calorimeter was also turned on. All detector subsystems functioned very well and the detector started science data collection on Dec. $30^{\text {th }} 2015$, less than 2 weeks after the launch.

Thanks to the robust design, and the sun-synchronous orbit, the temperatures of the STK ladders are very stable, with a daily variation much less than $1^{\circ} \mathrm{C}$ (Figure 4 left). The slow monthly variation, maximum $\pm 2.5^{\circ} \mathrm{C}$, is due to the orientation of the satellite orbit, moderated by the Earth's shadow from May to July. The temperature of the ladders follows closely that of the radiators (Figure 4 right), which indicates that the thermal management system of the STK functions properly.
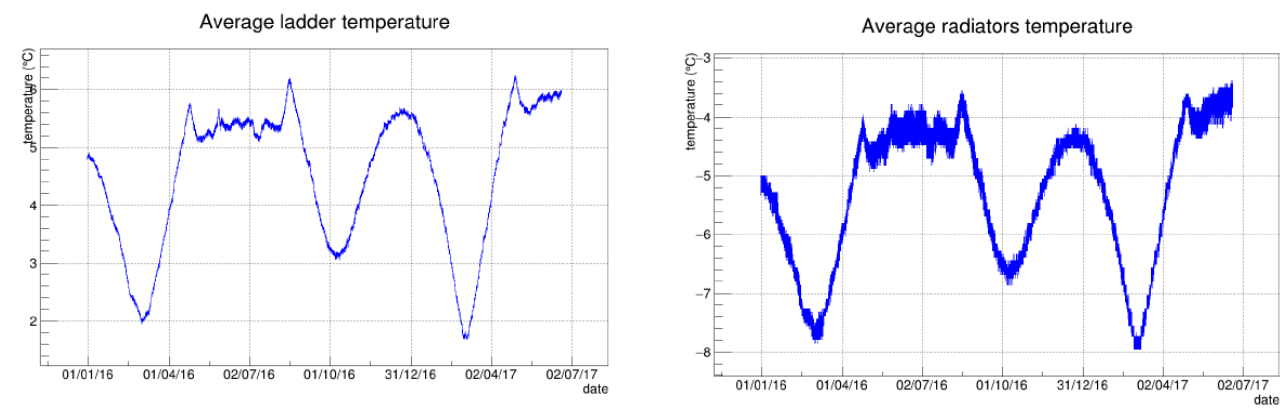

Figure 4. The average ladder temperature (Left) and the average radiator temperature (Right) as a function of time since launch.

A good temperature stability leads to a good noise stability: the variation of the average noise of all 74k channels (Figure 5 left) is within 0.04 ADC counts since the beginning of the data taking. The stability of noise makes the in-orbit operation of the STK easier, eliminating the need of the complex and time consuming operation of updating from the ground on-board 
zero suppression thresholds when noise changes. The average noise is $\sim 2.85 \mathrm{ADC}$, while the MIP signal for a particle hitting a readout(floating) strip is 52(27) ADC (note the charge measurement of the STK is reported in another paper [4] in this proceedings). Another indicator of the excellent quality of the STK is the fraction of good channels (conservatively defined as noise $<5$ ADC). It started out at $99.55 \%$ at the beginning of data taking, and improved as a function of time due to the stabilization in space, to $99.71 \%$ in July 2017 (green points in Figure 5 right).
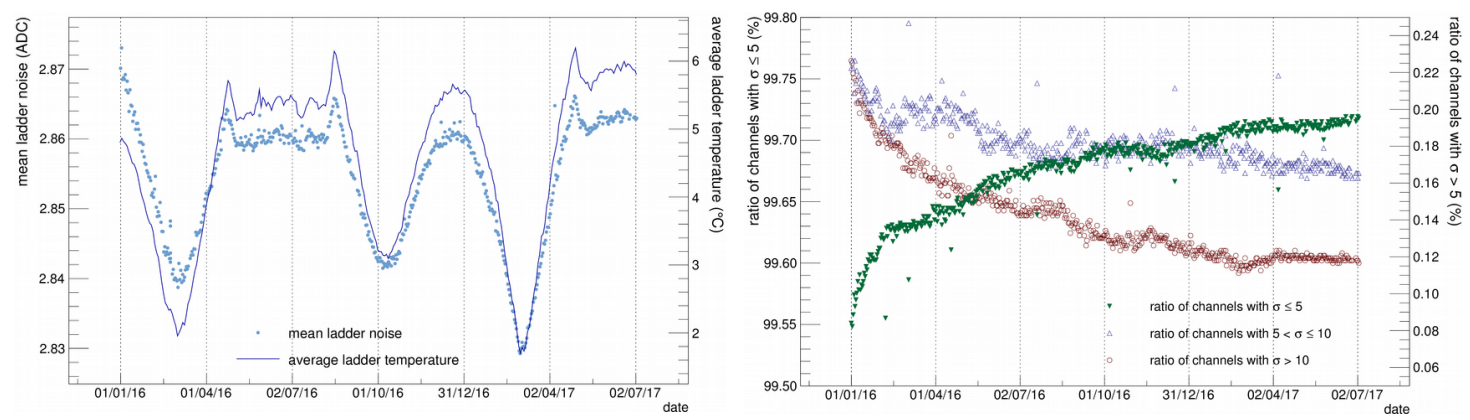

Figure 5. (Left) The average ladder temperature and the average STK channels noise; (Right) Fraction of STK channels with noise below 5 (green), between 5 and 10 (blue), and above 10 ADC (red).

The good temperature stability also makes the STK mechanically stable. Nevertheless to achieve the best possible position resolution provided by the detector, even the small misalignments caused by the long term temperature variation need to be corrected. This is done with an alignment procedure performed every 2-weeks with in-orbit data. This is sufficient to keep the tracker aligned for track reconstruction. As shown in Figure 6, after alignment, the measured position resolution is $\sim 40 \mu \mathrm{m}$ for the inner layers, where the extrapolation error is small. This is much better than the $75 \mu \mathrm{m}$ required.
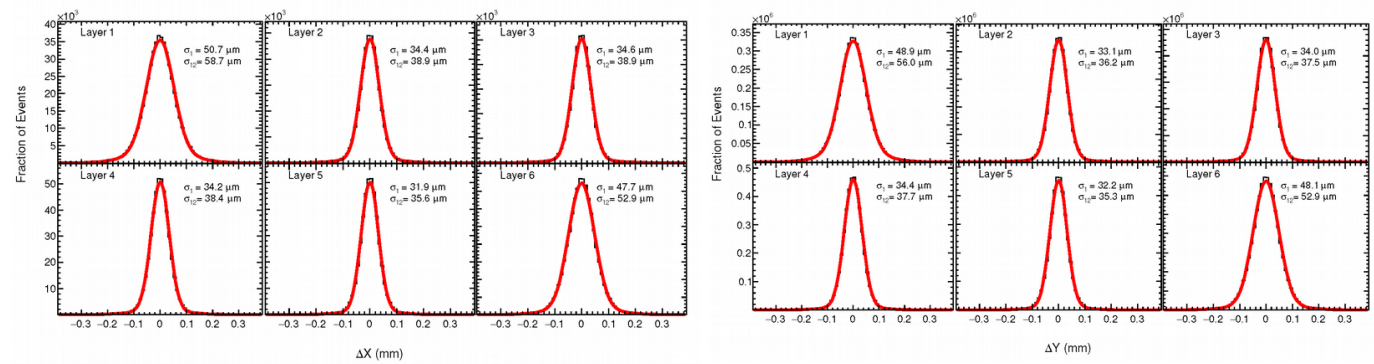

Figure 6. Unbiased hit residual distribution of $6 x$-layers (left) and $6 y$-layers (right) after alignment measured with in-orbit data, with double Gaussian fits. Tracks of all inclinations within the STK acceptance $\left(<60^{\circ}\right)$ are used.

The importance of the alignment is demonstrated in Figure 7 (left panel), where the effective position resolutions (RMS of a double Gaussian fit to the unbiased residuals of track-hit in a layer obtained using Minimum Ionizing Particles from in-orbit data) of all 12 STK layers, as a function of the incidence angle, before and after alignment, and compared to the simulation with perfect alignment. The alignment improve greatly the position resolution, reaching in most cases within $10 \%$ of the perfect alignment. The effect of the time dependent alignment is shown in Figure 7 (right panel), by comparing the RMS of the major Gaussian fit of the residual, normalized to January $1^{\text {st }} 2016$, as a function of time, with and without time dependent alignment (once every 2 weeks), for 2 incidence angular ranges. It can 
be seen that without time dependent alignment, the resolution of vertical entry tracks $\left(0^{\circ}-20^{\circ}\right)$ would be worsen by a few percent in 6 months, while inclined tracks $\left(>50^{\circ}\right)$ would have their position resolutions worsen by up to $30 \%$.

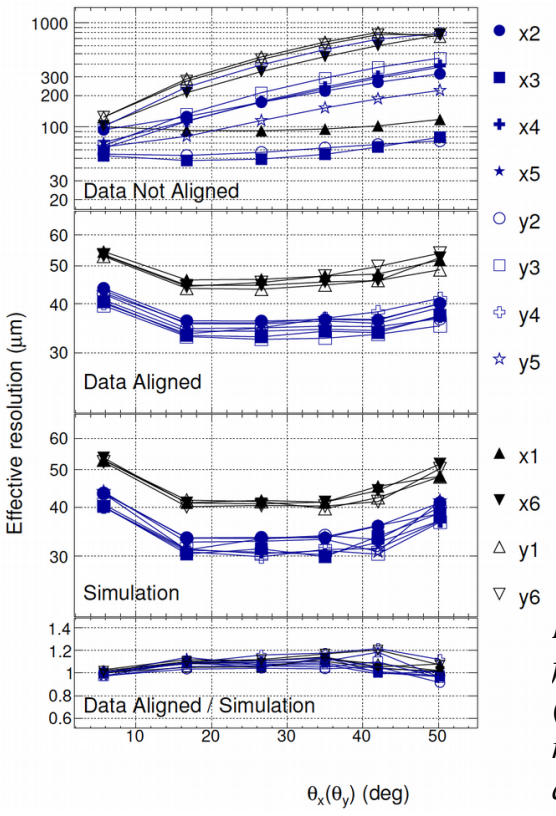

The a Kalman filter [5] based track finding algorithm, which is particularly suited for a tracker with substantial material, has been implemented for the STK and tuned with both Monte Carlo (MC) simulation events and in-orbit data. The realistic detector geometry model needed for the Kalman filter, including all sensitive volumes and the supporting structures of the payload, has been imported into the simulation and reconstruction software from ComputerAided-Design (CAD) drawings, using a conversion software tool-kit [6]. After several iterations of tuning a high track finding efficiency has been achieved for electrons and light nuclei (proton and Helium). Further optimization to improve tracking efficiency for heavy nuclei is in progress. Figure 8 shows the track finding efficiency for events passed a particular set of electron selection cut, as a function of the reconstructed energy in the calorimeter. A tracking efficiency of $\sim 97 \%$, constant in energy over a large range, has been achieved, and the agreement between data and $\mathrm{MC}$ is $\sim 1 \%$. Note a main contribution to the inefficiency is due to the insensitive areas between silicon sensors.

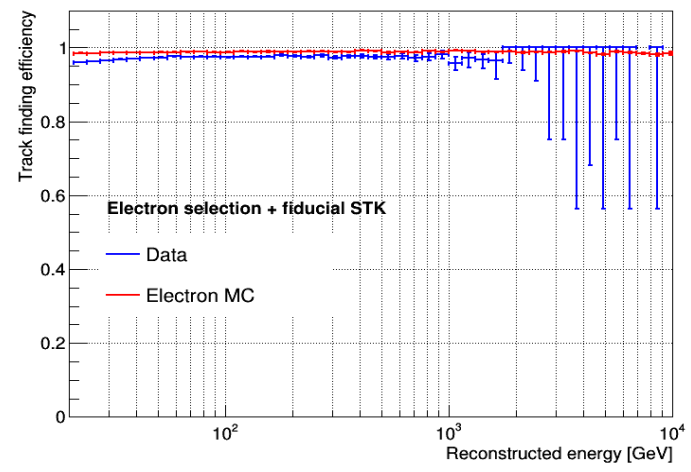

Figure 8: Track finding efficiency for electrons within the STK fiducial volume, as a function of the reconstructed energy in the calorimeter, for data (blue) and electron MC simulation (red). 


\section{Conclusions}

The Silicon-Tungsten Tracker (STK) of the DAMPE mission is based on robust technology of single-sided silicon strip detectors with analog readout. It plays crucial roles in charge track reconstruction, gamma-ray detection, cosmic ray charge measurement, and overall particle identification. After the successful launch of the DAMPE satellite, the STK has been commissioned quickly and has shown excellent noise, thermal and mechanical stabilities. Inorbit calibration and alignment procedures have been implemented and validated, allowing to achieve the best possible position and angular resolutions and long term stability. The track reconstruction algorithm has achieved a high track finding efficiency for electron and light nuclei (proton and Helium).

\section{Acknowledgement}

This work is supported by the Chinese Academy of Sciences, the Swiss National Science Foundation and INFN, Italy. The support of CERN for providing beam time allocation and technical assistance at the PS and SPS beam lines, as well as general logistical and technical support, including the bonding of several ladders, is acknowledged.

\section{References}

[1] J. Chang, J. et al. (The DAMPE collaboration). The Dark Matter Particle Explorer mission. Astropart. Phys., submitted (2017).

[2] P. Azzarello et al., Nuclear Instruments and Methods in Physics Research A 831 (2016) 378-384

[3] V. Gallo et al., Proceedings of the 34th International Cosmic Ray Conference (The Hague, Holland, 2015) PoS(ICRC2015)1199.

[4] S. Vitillo et al., Measurement of cosmic ray charge with DAMPE Silicon-Tungsten Tracker, this proceedings.

[5] R. Fruhwirth, Application of Kalman filtering to track and vertex fitting, Nucl. Instrum. Meth. A262 (1987) 444-450.

[6] A. Tykhonov, et al., Software framework and the reconstruction software of the DAMPE gammaray mission, in proceeding of 34th ICRC 1193. 